Relations industrielles

Industrial Relations

\title{
Globalization and the Future of Labour Law, sous la direction de John D.R. CRAIG et S. Michael LYNK, Cambridge, U.K. : Cambridge University Press, 2006, 498 p., ISBN-13 : 978-0-521-85490-0.
}

\section{Pierre Verge}

Volume 62, numéro 3, été 2007

URI : https://id.erudit.org/iderudit/016494ar

DOI : https://doi.org/10.7202/016494ar

Aller au sommaire du numéro

Éditeur(s)

Département des relations industrielles de l'Université Laval

ISSN

0034-379X (imprimé)

1703-8138 (numérique)

Découvrir la revue

Citer ce compte rendu

Verge, P. (2007). Compte rendu de [Globalization and the Future of Labour Law, sous la direction de John D.R. CRAIG et S. Michael LYNK, Cambridge, U.K. :

Cambridge University Press, 2006, 498 p., ISBN-13 : 978-0-521-85490-0.]

Relations industrielles / Industrial Relations, 62(3), 571-573.

https://doi.org/10.7202/016494ar

Tous droits réservés (C) Département des relations industrielles de l'Université Laval, 2007
Ce document est protégé par la loi sur le droit d'auteur. L'utilisation des services d'Érudit (y compris la reproduction) est assujettie à sa politique d'utilisation que vous pouvez consulter en ligne.

https://apropos.erudit.org/fr/usagers/politique-dutilisation/ 
le seul reproche que l'on peut faire à cet ouvrage original, tant au plan de son approche méthodologique qu'à celui des orientations qu'il propose pour repenser le droit des rapports collectifs de travail.

GuYlaine ValléE

Université de Montréal

\section{Globalization and the Future of Labour Law,}

sous la direction de John D.R. CRAIG et S. Michael LYNK, Cambridge, U.K. :

Cambridge University Press, 2006, 498 p., ISBN-13 : 978-0-521-85490-0.

Sous ce titre accrocheur, qui relie l'accentuation contemporaine du phénomène de mondialisation et l'avenir du droit du travail, se regroupent quinze études initialement présentées lors d'un symposium à l'Université de Western Ontario, London Ontario, en octobre 2003. Les auteurs, en quasi-totalité, sont des juristes, praticiens, universitaires ou fonctionnaires internationaux. Nous faisons d'abord simplement état de ces textes selon la répartition, parfois contestable et en six parties, choisie par les directeurs de l'ouvrage, MM. Craig et Link; nous tenterons ensuite de voir si certaines observations fondamentales communes se dégagent de ces études, pour finalement apprécier le tout.

La partie I veut apporter certaines perspectives sur la mondialisation. (Une tentative d'appréhender systématiquement ce phénomène lui-même devra toutefois attendre un texte de la partie suivante (Véronique Marleau, p. 110).) Si l'une de ses deux composantes, soit l'étude du professeur Harry Arthurs, insiste sur certains éléments de ce phénomène, comme l'impact des entreprises transnationales, elle apporte surtout une description de changements induits par ce dernier, non pas tant dans le contenu que dans les modes d'application du droit du travail canadien. Quant à l'autre texte initial (Brian Burkett), il brosse un tableau général de la normativité internationale du travail, à la fois son contenu substantiel et institutionnel (OIT, Union européenne, ALENA, MERCOSUR, Sommets des Amériques).
La partie suivante se voulait précisément consacrée aux « normes internationales du travail ». Kevin Banks y démontre essentiellement que les normes du travail élevées dans un pays et avantage économique pour ce dernier sur la scène internationale sont en synchronie. Véronique Marleau préconise ensuite, relativement à l'adaptation aux incidences de la mondialisation sur l'organisation de la production, de s'en remettre au principe dit de subsidiarité, inspiré de l'Union européenne, pour ce qui est de la détermination des liens d'élaboration des normes du travail, en particulier l'encadrement de la négociation collective. La fragmentation territoriale qui caractérise la formule courante à cet égard au Canada paraît ainsi partiellement anachronique (p. 119). Enfin, Alan Hyde fait appel à la théorie économique des jeux pour justifier les standards internationaux du travail, particulièrement ceux issus d'ententes bilatérales ou régionales.

La partie III est consacrée à l'Union européenne. Manfred Weiss expose d'abord les difficultés d'adaptation des droits du travail des pays de l'Europe centrale qui se sont joints à l'Union en 2004 au regard des normes et des institutions de cette dernière, en particulier sa culture du dialogue social; Arturo Bronstein brosse ensuite un tableau de l'évolution de ces droits nationaux du travail, de l'ère socialiste à celle du marché. Catherine Barnard complète ce volet européen en se concentrant spécifiquement sur 
le droit relatif à la sécurité sociale des travailleurs migrants au sein de l'Union. Une quatrième partie nous fait passer aux Amériques. Lance Compa, après avoir exposé l'essentiel des interventions subrégionales relatives au travail (Accord nord-américain de coopération en matière de travail (ANACT) et autres ententes plus ou moins similaires liant les États-Unis et le Canada, institutions du travail du MERCOSUR, dont la Declaración sociolaboral, et celles de la CARICOM), suggère, dans une perspective d'avenir, de bâtir à partir de ces acquis et de leur influence particulière sur l'effectivité des droits nationaux du travail en cause; en effet, une approche supranationale ne serait pas réaliste dans le contexte des Amériques (p. 265). Brian Langille apporte ensuite une réflexion qui, bien que présentée en fonction d'un désormais plus qu'éventuel projet de libre-échange des Amériques, est de portée plus vaste; il conclut à la nécessité s'assurer les droits fondamentaux du travail pour en arriver à une stratégie de développement économique et social à l'avantage des pays qui se rapprochent; de là, un accent sur des programmes d'assistance, par opposition aux sanctions et à l'exclusion pour réaliser un tel objectif (p. 297). Jose Pastore nous fait enfin pénétrer dans le contexte socio-économique des pays du MERCOSUR et plus généralement de l'Amérique latine, où notamment $60 \%$ de la force du travail se rattache au secteur informel. Il examine aussi, non sans un certain scepticisme, les institutions du travail de ce MERCOSUR, de même le contenu général des droits du travail de ses pays, droits empreints souvent de rigidité et pas toujours effectifs, selon l'auteur. S'inspirant notamment de l'ANACT, ce dernier recherche une solution d'avenir basée sur la coopération régionale, par opposition aux sanctions commerciales, pour assurer, en fait, ces droits du travail déjà substantiellement explicites (p. 323).
Une cinquième partie est consacrée aux normes de l'OIT. Werner Singenberger réfute l'argumentaire économique à leur encontre et défend leur universalité; leur mise en œuvre, la confrontation EstOuest disparue, requiert la concertation d'acteurs non seulement étatiques, mais aussi d'autres issus de la société civile (p. 353). Edward Potter repère enfin des situations particulières d'appropriation du droit international du travail, dans ses aspects fondamentaux, dans certaines espèces judiciaires aux États-Unis, de même qu'un renvoi à la Déclaration de 1998 de l'OIT dans les accords commerciaux internationaux récemment signés par ce pays. Une sixième et dernière partie, intitulée simplement «Labour Rights » relève deux aspects spécifiques, mais sensibles, de ce thème général : l'affirmation des droits de la femme au travail dans le droit international du travail et la protection des droits des travailleurs migrants par ce droit. Dans le premier cas, Mary Cornish, Fay Faraday et Verna Verma décrivent non seulement l'évolution des instruments pertinents de l'ONU, de l'OIT, de même que ceux se rattachant à différents ensembles régionaux, mais d'abord et avant tout les difficultés concrètes de réalisation du principe d'égalité à l'endroit des femmes au travail (p. 389); dans le second, Ryszard Cholewinski fait notamment état du peu de ratification des instruments internationaux régissant la mobilité internationale des travailleurs et suggère différentes initiatives pour assurer les droits de ces travailleurs souvent vulnérables (p. 433).

Nombreux sont les aspects soulevés dans un ensemble aussi diversifié d'études et dont le contenu n'a pu être évoqué ici que d'une manière incomplète. Certains traits communs, en certain consensus, paraissent néanmoins s'en dégager. En premier lieu, est perceptible, une tendance à privilégier une approche «horizontale », c'est-àdire de collaboration entre États qui se 
regroupent dans des ensemble régionaux; le droit supranational continuera vraisemblablement à demeurer l'apanage de l'Union européenne (voir ainsi Burkett, Arthurs, Compa, Marleau). La normativité du travail de l'OIT semble aussi vouée à un rôle relativement plus effacé qu'à l'époque de l'après-guerre. Les droits nationaux (malgré les limites qu'entraîne leur territorialité, ce que l'on n'analyse pas tellement) conservent tous, leur importance. Ils doivent être effectifs; leur adaptation au contexte contemporain de mondialisation conduit à se soucier non seulement de leur contenu substantiel, mais aussi des institutions qui assurent sa mise en œuvre. L'établissement et l'application de normes du travail adéquates font partie de projets de société plus englobants et vont de pair, en longue portée, avec la prospérité économique des pays auxquels ils correspondent (voir ainsi Banks, Langille, Pastore). La coopération multiétatique, beaucoup plus que le recours à la sanction économique, assure l'atteinte de tels objectifs de la part de pays qui se rapprochent économiquement (ibid.). Tout comme les normes internationales du travail elles-mêmes, cette action multinationale doit également compter sur la participation d'acteurs de la société civile; groupements syndicaux, entreprises transnationales, ONG (voir ainsi Pastore, Singenberger, Cornish et al.).

Il revenait sans doute aux directeurs de l'édition de l'ouvrage, qui en avaient fort bien présenté et situé initialement le contenu, d'en dégager finalement un certain bilan. Tel complément eût distingué l'entreprise de plusieurs autres collectifs du jour tournant autour du thème "mondialisation-travail ». On peut aussi regretter que certaines des études de l'ouvrage n'explicitent pas de façon plus marquée et détaillée comment ce dit phénomène de mondialisation modifie l'organisation de la production, à laquelle celle du travail est ellemême liée. Le droit du travail, tant international que national, doit, en effet, directement correspondre à cette réalité productive transformée. Il n'en demeure pas moins que l'ouvrage réunit dans une facture impeccable un ensemble de contributions d'une grande richesse en la matière.

\section{Pierre Verge}

Université Laval

\section{Mobsters, Unions and Feds: The Mafia and the American Labor Movement,}

by James B. JACOBS, New York: New York University Press, 2006, 352 pp., ISBN-13: 978-0-8147-4273-0.

This book is about the connection between organized labour and organized crime in the United States. It addresses: misappropriation of worker pension and operating funds by union office holders and other criminals; extortion of employers by union officials and the mafia; and union/employer conspiracies to operate cartels that corruptly allocate contracts and set prices. It mostly deals with its topic, labour racketeering, historically. In taking this approach, the author analyses the enduring patterns of criminal behaviour of a small group of stakeholders in the labour movement. Underlying this discussion are portraits of distinct periods in $20^{\text {th }}$ century American history. These include: the bootlegging era; the congressional hearings "high-point" period of 195759 during which the McClelland senate select committee saw a procession of union officials and members of the mafia take the $5^{\text {th }}$ Amendment; the age of federal law enforcement's obsession with communism as the only matter worth investigating and the consequent unabated proliferation of criminal 